\title{
Physiological Aging as an Infinitesimally Ratcheted Random Walk
}

\author{
Bernardo A. Melld* \\ Institute of Physics, University of Brasilia, 70919-970, Brasilia, DF, Brazil
}

\begin{abstract}
The distribution of a population throughout the physiological age of the individuals is very relevant information in population studies. It has been modeled by the Langevin and the FokkerPlanck equations. A major problem with these equations is that they allow the physiological age to move back in time. This paper proposes an Infinitesimally ratcheted random walk as a way to solve that problem. Two mathematical representations are proposed. One of them uses a non-local scalar field. The other one is local, but involves a multi-component field of speed states. These two formulations are compared to each other and to the Fokker-Planck equation. The relevant properties are discussed. The dynamics of the mean and variance of the population age resulting from the two proposed formulations are obtained.
\end{abstract}

Published as: PHYSICAL REVIEW E 82, 021918 (2010)

DOI: 10.1103/PhysRevE.82.021918

PACS numbers: 87.23.Cc, 05.40.Fb, 87.10.Ed, 87.18.Tt

\section{INTRODUCTION}

The mathematical study of populations started with Malthus [1], who, in 1789, declared that the human population grows exponentially. Verhulst realized, in 1838, that the limited availability of resources prevents that growth and proposed the now famous logistic equation [2]. Further progress was made with the predator-prey equations, independently proposed by Lotka and Volterra [3, 4] in 1925 and 1926.

In these formulations, each species is described by one variable that represents the total number of individuals. It is often necessary to have information about the age structure of the population. In 1945 Leslie used a matrix containing the probability of surviving or reproducing until the next time step [5]. When that matrix is applied to a vector describing the number of individuals at each discrete age, the vector with the population at the next time step is obtained.

McKendrick [6], in 1926, and Von Forest 7], in 1959, expressed age by using a continuous variable. The age structure at time $t$ is replaced by the probability density $\hat{p}(a, t)$ where $a$ is the chronological age (c-age), i.e., the time elapsed from the birth of the individuals and the instant $t$. The dynamics of a population with a mortality rate $\hat{\kappa}(a)$ is described by the convective equation

$$
\frac{\partial \hat{p}(a, t)}{\partial t}=-\frac{\partial \hat{p}(a, t)}{\partial a}-\hat{\kappa}(a) \hat{p}(a, t) .
$$

Determining the c-age is not possible in several practical situations. In these cases, the population is divided into development classes depending on some characteristics of the individuals. In spite of the fact that these classes are related to the c-age, they are not an exact measurement of it. Since the sojourn time within a class

\footnotetext{
* bernardo@fis.unb.br
}

changes from one individual to another, Lefkovitch, in 1965, described survival and progression from one class to the next by a transition matrix, in a Markovian representation of aging [8].

The interaction of the individuals with the environment, among themselves and with other species depends on the physiological aspects measured by the physiological age $(p$-age, $\phi)$, through which they are related to the c-age. For that reason VanSickle, in 1977, proposed using the p-age to describe the population [9]. The p-age $\phi$ can be represented, for example, by the individual's weight or size, whose average increases monotonically with the c-age $a$. With the relation between these variables described by $\phi=\Phi(a)$, VanSickle rewrote Eq. (1) as

$$
\frac{\partial p(\phi, t)}{\partial t}=-\frac{\partial v(\phi) p(\phi, t)}{\partial \phi}-\kappa(\phi) p(\phi, t)
$$

where

$$
\begin{aligned}
v(\phi) & =\left.\frac{d \Phi}{d a}\right|_{\Phi^{-1}(\phi)}, \\
p(\phi, t) & =\frac{\hat{p}\left(\Phi^{-1}(\phi), t\right)}{v(\phi)}, \text { and } \\
\kappa(\phi) & =\hat{\kappa}\left(\Phi^{-1}(\phi)\right) .
\end{aligned}
$$

Two important measures of the population distribution are the mean value of $\phi$,

$$
\mu(t) \equiv \int \phi p(\phi, t) d \phi,
$$

and the variance of $\phi$

$$
\sigma^{2}(t) \equiv \int(\phi-\mu)^{2} p(\phi, t) d \phi
$$

with $p(\phi, t)$ normalized. None of the above approaches has a parameter that allows to fit the model to the experimentally measured variance. 
The variability of the development rate among individuals was introduced by Lee et al in 1976 [10]. After that, much work was done with the individual-based model of Huston, deAngelis and Post 11]. Kirkpatrick [12], Clother and Brindley [13] exploited the Ito equation with a Gaussian noise with mean zero, best known among physicists as the Langevin equation. In [14], Plant and Wilson studied populations with continuous dynamics within stages and discontinuous stage structures. A formulation of this model using partial derivatives, the Fokker-Planck equation, was utilized by Buffoni and Pasquali [15]

$$
\frac{\partial p(\phi, t)}{\partial t}=-v \frac{\partial p(\phi, t)}{\partial \phi}+\frac{D}{2} \frac{\partial^{2} p(\phi, t)}{\partial \phi^{2}} .
$$

The time evolution of the p-age depends on several environment factors. The metabolic rates of insects, for example, change with the temperature. For that reason the variables $v$ and $D$ in Eq. (8) could be functions of the temperature, $T$. Despite this fact, they will be kept constant from now on.

A method that has being applied in practical situations is the calculus of degree-days [16] between the occurrence of a biofix (a detectable biological event) at time $t_{0}$ and the development stage at time $t_{1}$. Formally it is given by

$$
D D \equiv \int_{t_{0}}^{t_{1}}\left(T(t)-T_{0}\right) H\left(T(t)-T_{0}\right) d t
$$

where $H(x)$ is the Heaviside step function, $T$ is the environment temperature and $T_{0}$ is the baseline temperature. Since the development rate is believed to be approximately proportional to $T-T_{0}$, the number of degree-days is proportional to the physiological development. The peak of an infestation is used as the biofix and the evolution of the population surge is evaluated by the integral. It is, notwithstanding, not a population description, but just an estimate of the development state of a population sample.

The p-age can also be an abstract indicator of the individuals' maturity [15]. That description is particularly suitable for species with well-defined stages [17]. Although that abstract value cannot be determined for every individual, specific values of the p-age are related to unmistakable biological events, such as eggs hatching, emergence from pupae, etc. Since the Fountain of Youth has not yet been discovered, it makes no sense to allow p-age to move backward. Even if such backward movements could happen at molecular level, they are forbidden at the thermodynamic limit represented by the page of an individual. Therefore, the Langevin and the Fokker-Planck equations are inappropriate descriptions.

A more suitable mathematical model would be a continuous variable that moves forward with steps of random, positive length. The physical model for such a system is an infinitesimally ratcheted random walk (IRRW). It is not a Brownian ratchet in the sense introduced by
Feynman [18], because, in the present approach: a) backward steps are completely forbidden, not just less probable; $b$ ) the ratchet teeth are infinitesimal, meaning that even the smallest movement advances the ratchet to another tooth; $c$ ) no reference is made to mechanics or the thermodynamics theory.

Two mathematical models for the IRRW are presented below, disregarding the boundary effects, reproduction, and death, which results in probability conservation. As will be shown, in both models the values of $\mu$ and $\sigma^{2}$ evolve uniformly on time:

$$
\frac{d \mu(t)}{d t}=v \quad \frac{d \sigma^{2}(t)}{d t}=D
$$

In each of the models proposed, expressions for $v$ and $D$ are found.

\section{NON-LOCAL FORMULATION}

The simplest approach to the IRRW involves the scalar field $p(\phi, t)$. Since we are not concerned with interparticle interactions, the equation must include linear terms only. Furthermore, the dynamics must be translationally invariant on $\phi$, at least inside a given development stage. The most general equation satisfying these requirements is

$$
\frac{\partial p(\phi, t)}{\partial t}=-\alpha p(\phi, t)+\alpha \int p\left(\phi^{\prime}, t\right) f\left(\phi-\phi^{\prime}\right) d \phi^{\prime} .
$$

Conservation imposes

$$
\int f(\phi) d \phi=1
$$

The Fokker-Planck equation corresponds to having $\alpha=1$ and

$$
f(\phi)=\delta(\phi)+v d \frac{\delta(\phi)}{d \phi}-D \frac{d^{2} \delta(\phi)}{d \phi^{2}} .
$$

The space-discretized form of Eq. (11) is

$$
\frac{d p_{j}(t)}{d t}=-\alpha p_{j}+\alpha \sum_{k} p_{k}(t) f_{j-k},
$$

with $f_{k}=f(k \Delta x) \Delta x$. A form of the Fokker-Planck equation accurate up to the second order in space can be written with $f_{k}=0$ for all $k \notin\{-1,0,1\}$ [19]. On the other hand, the IRRW implies in $f_{k} \geq 0$ for $k \geq 0$ and $f_{k}=0$ for $k<0$. Similarly, it requires

$$
\begin{cases}f(\phi) \geq 0 & \text { for } \phi \geq 0 \\ f(\phi)=0 & \text { for } \phi<0\end{cases}
$$

The above conditions exclude the Fokker-Planck equation or any form of Eq. (8) involving $\phi$ derivatives. 
By substituting Eq. (11) in the time derivative of equations (6) and (7) we can describe the evolution of the average and variance of the p-age as

$$
\frac{d \mu(t)}{d t}=\alpha\langle x\rangle_{f}, \quad \frac{d \sigma^{2}(t)}{d t}=\alpha\left\langle x^{2}\right\rangle_{f}
$$

where

$$
\langle g(x)\rangle_{f}=\int g(x) f(x) d x .
$$

The comparison of these equations with Eq. (10) leads to the conclusion that $\alpha$ and the function $f(x)$ must be such that

$$
v=\alpha\langle x\rangle_{f} \quad D=\alpha\left\langle x^{2}\right\rangle_{f} .
$$

Whatever function $f(x)$ is, if the values of $\langle x\rangle_{f}$ and $\left\langle x^{2}\right\rangle_{f}$ are definite, it is always possible to rescale $f(x)$ and choose $\alpha$ so that the above equations are satisfied.

If $f(x)$ doesn't include delta functions centered in 0 then $f(x) \neq 0$ for values of $x \neq 0$ and it must, according to Eq. (18), extends for at least a finite $L>0$. Consequently, the IRRW represented by Eq. (11) covers finite distances in an infinitesimal time interval, meaning that the particles have infinite speed. Although this fact may seem strange, it should not be a serious problem, since even the well-accepted Fokker-Planck equation presents that property.

Similar use of an integral can be find in [20], where the usual nonlinear term of the Fisher equation was integrated over space to express nonlocal competition. In our model, the integral is over the physiological age, but it is still referred to as the nonlocal term.

The integral form (11) is not suitable for numerical calculations, since an integral must be evaluated at each discrete point $\phi$. Fortunately, there exist functions for which the integral at $\phi$ may be quickly calculated from the value at $\phi-\Delta \phi$. Two of these functions are the linear and exponential functions.

\section{SPEED STATES FORMULATION}

Besides using a non-local formulation and a scalar field, the IRRW can be described by a multi-component field $\mathbf{p}(\phi, t)=\left\{p_{1}(\phi, t), \cdots, p_{n}(\phi, t)\right\}$ with the probability density given by

$$
p(\phi, t)=\sum_{i} p_{i}(\phi, t) .
$$

Each component $p_{i}$ corresponds to a population that moves without dispersion with speed $v_{i}$, and switch from state $i$ to state $j$ with rate $T_{j i}$. They are subject to a local dynamic equation

$$
\frac{d p_{i}(\phi, t)}{d t}=-v_{i} \frac{d p_{i}(\phi, t)}{d \phi}+\sum_{j} T_{i j} p_{j}(\phi, t) .
$$

If the above equation are integrated on $\phi$ disregarding the boundary effects, the master equation is found to be

$$
\frac{d p_{i}(t)}{d t}=\sum_{j} T_{i j} p_{j}(t)
$$

where

$$
p_{i}(t) \equiv \int p_{i}(\phi, t) d \phi
$$

Probability conservation imposes $\sum_{i} T_{i j}=0$. By summing Eq. (20) over $i$ we arrive to the dynamics equation for the population density

$$
\frac{\partial p(\phi, t)}{\partial t}=-\sum_{i} v_{i} \frac{\partial p_{i}(\phi, t)}{\partial \phi}
$$

Being $\mathbf{T}$ a Markov matrix for continuous time, its eigenvalues are all negative, except by one nondegenerated eigenvalue $\lambda^{0}=0$. The components $p_{i}^{0}$ of this stationary normalized eigenvector are all greater than or equal to zero. If $\lambda^{1}$ is the second greatest eigenvalue, $\mathbf{p}\left(t \gg 1 /\left|\lambda^{1}\right|\right) \approx \mathbf{p}^{\mathbf{0}}$.

Even after the steady state of Eq. (21) is reached, the convective term of Eq. (20) constantly moves the local population away from the equilibrium. After the transient is gone, the stationary state $\mathbf{p}(t)=\mathbf{p}^{\mathbf{0}}$ can be used to derive the dynamics of

$$
\mu_{i}(t) \equiv \frac{1}{p_{i}^{0}} \int \phi p_{i}(\phi, t) d \phi
$$

from Eq. (20), resulting in

$$
\begin{aligned}
\frac{d \mu_{i}(t)}{d t} & =v_{i}+\frac{1}{p_{i}^{0}} \sum_{j} T_{i j} p_{j}^{0} \mu_{j}(t) \\
& =v_{i}+\frac{1}{p_{i}^{0}} \sum_{j} T_{i j}^{0} \mu_{j}(t) .
\end{aligned}
$$

Since $\mathbf{T}$ is a continuous time Markov matrix, $T_{i j}^{0} \equiv T_{i j} p_{j}^{0}$ share the same property. Furthermore, once $\mathbf{p}^{\mathbf{0}}$ is an eigenvector of $\mathbf{T}$ with eigenvalue 0 , a vector with all elements identical will be an eigenvector of $\mathbf{T}^{\mathbf{0}}$ with the same eigenvalue.

In this stationary regime, the dynamics of

$$
\mu(t)=\sum_{i} p_{i}^{0} \mu_{i}(t)
$$

can be derived from Eq. (25) or by integrating Eq. (23):

$$
\frac{d \mu(t)}{d t}=\sum_{i} v_{i} p_{i}^{0} \equiv v^{0}
$$

The solution of that equation is

$$
\mu(t)=m^{0}+v^{0} t .
$$


The unceasing exchange of particles among the speed states at Eq. (20) connects the population of these states in bundles moving with speed $v^{0}$.

The motion of the center of mass of each state depends not only on its own speed but also on the other states' centers of mass positions. The motion is governed by the nonhomogeneous Eq. (25) which possesses the particular solution

$$
\mu_{i}(t)=m_{i}+v^{0} t,
$$

with $m_{i}$ satisfying

$$
\frac{1}{p_{i}^{0}} \sum_{j} T_{i j}^{0} m_{j}=v^{0}-v_{i}
$$

The homogeneous part of Eq. (25),

$$
\frac{d \mu_{i}(t)}{d t}=\frac{1}{p_{i}^{0}} \sum_{j} T_{i j}^{0} \mu_{j}(t)
$$

depends on the eigenvalues of $T_{i j}^{0} / p_{i}^{0}$. The eigenvalues of $\mathbf{T}^{\mathbf{0}}$ are all negative but the zero one. The same property is shared by $T_{i j}^{0} / p_{i}^{0}$ because $p_{i}^{0}>0$. The homogenous solution disappears after a while, except for the eigenvector $\mu_{i}=m^{0}$ of the null eigenvalue, which can be included in $m_{i}$ of eq. (29). Therefore, eq. (29) is the asymptotic solution of eq. (25).

Although the population average position at each speed state can be ahead or behind the average position of the whole group, they all move forward with the same average speed $v^{0}$. The null eigenvalue corresponding to the constant eigenvector of $\mathbf{T}^{\mathbf{0}}$ means that the vector $m_{j}=$ const can be added to the solution of (30), reflecting the translational invariance of the system.

After some algebra, the time evolution of $\sigma^{2}(t)$ can be obtained from Eq. (20) and (29) when $t \gg 1 /\left|\lambda^{1}\right|$. Thanks to this result, together with Eq. (27), expressions for $v$ and $D$ are found

$$
\begin{aligned}
v & =\sum_{i} v_{i} p_{i}^{0}, \\
D & =2 \sum_{i}\left(m_{i}-m^{0}\right) v_{i} p_{i}^{0} .
\end{aligned}
$$

The subtraction of $m^{0}$ from $m_{i}$ removes any dependence on a uniform translation related to the zero eigenvalue of $\mathbf{T}^{0}$.

The simplest possible system able to accommodate Eq. (32) is

$$
\mathbf{T}=\left[\begin{array}{cc}
-k & k \\
k & -k
\end{array}\right] \quad \mathbf{v}=\left[\begin{array}{l}
0 \\
\nu
\end{array}\right]
$$

The eigenvalues and eigenvectors of $\mathbf{T}$ are

$$
\begin{aligned}
\lambda^{0}=0, & \mathbf{p}^{\mathbf{0}}=\frac{1}{2}\left[\begin{array}{l}
1 \\
1
\end{array}\right], \\
\lambda^{1}=-2 k, & \mathbf{p}^{\mathbf{1}}=\frac{1}{2}\left[\begin{array}{c}
1 \\
-1
\end{array}\right] .
\end{aligned}
$$

When these values are substituted in Eq. (30) the values of $m_{i}$ are obtained (the two equalities of the linear system are not linearly independent)

$$
\mathbf{m}=\left[\begin{array}{l}
m^{0}-\nu / 4 k \\
m^{0}+\nu / 4 k
\end{array}\right]
$$

The average value of $\phi$ of the whole population in $t=0$ would be $m^{0}$, provided that equilibrium was already reached at that time. The average position of the zero speed individuals is behind the average population's position, while the $\nu$ speed individuals move ahead of the group.

Concluding, the constants for the two speed states system are

$$
v=\frac{\nu}{2} \quad D=\frac{\nu^{2}}{4 k} .
$$

\section{COMPARING THE MODELS}

The time evolution of $\mu(t)$ and $\sigma^{2}(t)$ obtained by numeric integration of the three models can be seen in figures $1 a$ and $1 b$. When $t \lesssim 1, \sigma^{2}(t)$ of the two speeds model slightly deviates from the linear behavior, which may be due to the transients of the homogeneous solution of Eq. (25). Except for that, these two curves perfectly agree with the analytic results.

Figure 22 $a$ shows that the three approaches result in very different population distribution for short time scales. In the Fokker-Plank dynamics the initial delta distribution becomes immediately Gaussian, while the disappearing of the delta takes some time in the other two models. Aiming to a quantitative evaluation of this phenomenon, we define the quantity

$$
q(t)=\int \delta(\phi) p(\phi, t) d \phi .
$$

The evolution of $q(t)$ can be seen in figure 1;.

The instantaneous disappearing of the delta function in the Fokker-Plank equation, resulting in $q(0) \approx 0$, is only possible due to the singularity of the laplacian of the delta function. The nonexistence of a similar singularity in the non-local model prevent the same effect in this model. In the two speeds model, the singular derivative of $d p / d \phi$ in the $v>0$ state instantly moves the population at this state away from $\phi=0$, resulting in $q(0)=1 / 2$.

If we apply the frame of reference change $x \rightarrow x-\nu t$ in the two states model, the only effect would be replacing $\mathbf{v}$ in eq. (34) by

$$
\mathbf{v}=\left[\begin{array}{c}
-\nu \\
0
\end{array}\right]
$$

The resulting distribution with such $\mathbf{v}$ would be translations of the figure 2 distributions. On the other hand, symmetry analysis implies that the distributions resulting from eq. (34) or from eq. (40) should be symmetric 

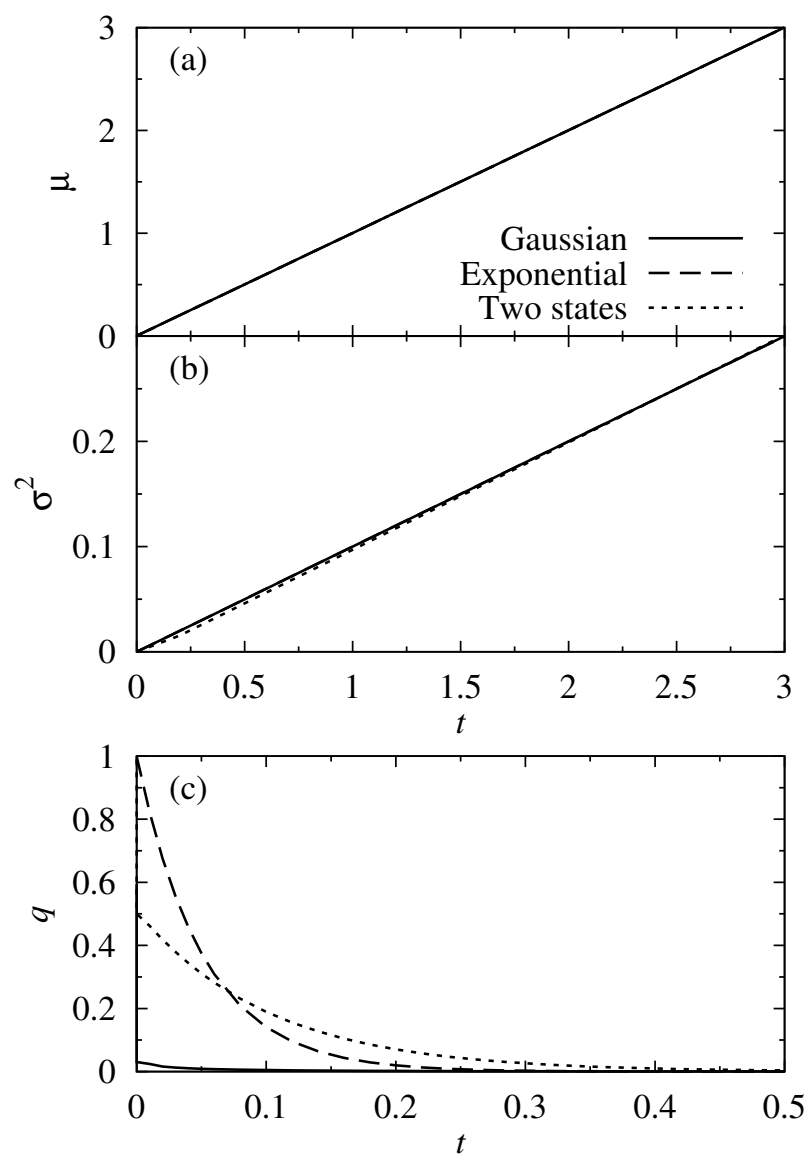

FIG. 1. Results of the numerical integration according to the Fokker-Plank equation (Gaussian), non-local IRRW with exponential function and two speed states model, with $v=1$ and $D=0.1$. The initial distribution was $p(\phi, 0)=\delta(\phi)$, with the population of the two speed model already in stead state: $p_{0}(t=0)=p_{1}(t=0)$. a) Population average position. b) Population position variance. c) Value of $q(t)$ as defined in Eq. (39).

by reflection, and a delta should be present at the right of the bells seen in figures $2 a$ and $2 b$. A trace of such delta function is present on figure 2a, but complectly disappeared on figure $2 b$, due to the numerical discretization.

The asymmetry of the distribution resulting from the non-local model is visible when we compare that distribution, in figure 2 with the perfectly symmetric the FokkerPlanck distribution. That asymmetry is not a surprise, since the non-local Eq. (11) is not symmetric.

After some time, the distribution at $\phi>0$ of the IRRW models look like a truncated bell, but the curves become bell shaped only after the distribution moves far enough from the initial position. The two states model becomes Gaussian faster than the non-local model. This happens despite the initial asymmetry generated in the two states model by the numerical error.

Regarding numerical implementation, the FokkerPlanck equation usually involves inverting a tri-diagonal
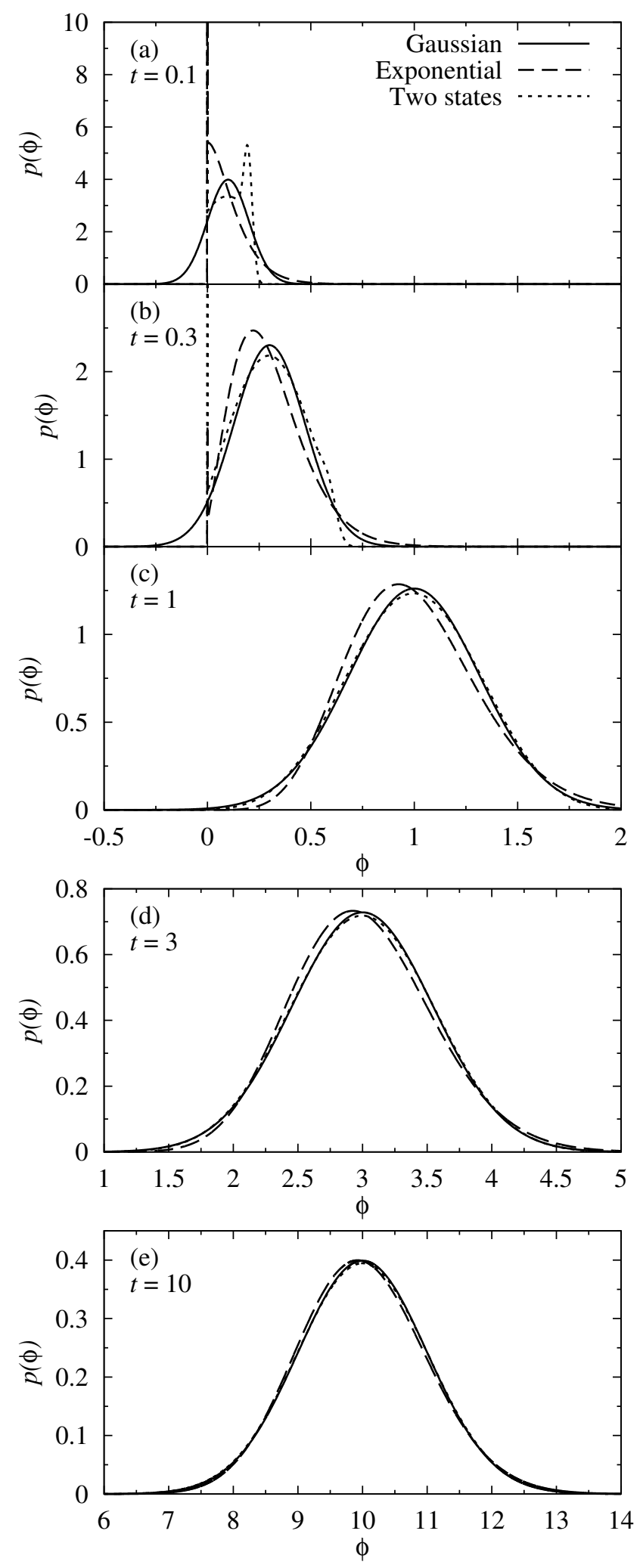

FIG. 2. Population distribution resulting from the same numerical integration of figure 1 at different instants for the three models. 
matrix when using second-order accuracy on time. The two speeds model methods doesn't, since it can be implemented using upwind differencing.

When applying the boundary conditions to the FokkerPlanck equation, it is necessary to prevent the movement in the wrong direction. One doesn't need to worry about such matters when using the IRRW models. On the other hand, it is awkward to include far-reaching effects of the non-local formulation in the boundary conditions, mainly between development stages.

Except for involving more than one field, the numerical implementation of the two speed states model is overall more convenient than the Fokker-Planck and the nonlocal models.

Only specially planned experiments, or a deeper understanding of the aging process, will decide which, if any, of the three models discussed in this work is correct. Whatever the answer is, it won't invalidate the use of the other methods as a convenient approximation, not to mention the fact that many different behaviors can be accommodated in the freedom of choosing $f(x)$ in Eq. (11) or $v_{i}$ and $T_{i j}$ in Eq. (20).

\section{CONCLUSION}

Two basic equations describing the evolution of the population distribution were proposed. As already mentioned, the main problem with the Fokker-Planck equation is its non-physical properties, namely, negative variation of $\phi$ and the infinite speed. The non-local formulation solves the first problem but not the second, while the speed states formulation solves both of them.

An advantage of the non-local and the two speed mod- els over the Fokker-Plank equation is their convenience for certain numerical implementations. The analytical expressions for the mean and the variance of the p-age help to understand the role played by the parameters of the dynamic equations.

Several other biological phenomena could be included in a more detailed formulation. Some of them, such as the age structure, the quiescence, and the dependence of the biological development on the temperature, could be included by making the equation parameters dependent on temperature and age. Other phenomena such as death, reproduction, and diapause, require the introduction of new terms in the dynamic equation. Introducing more complex effects such as spatiality and inter species interaction can only be done by defining new independent variables and fields.

Real situations demand taking into account some of these phenomena, requiring several additional assumptions and the determination of the corresponding parameters. Possible usages include plague control, epidemics, ecological management, demography, etc. They go well beyond the scope of this paper, which presents the equations ruling the intra-stage development.

Most models of genetic agents explored by physicists use c-age, either discrete 21] or continuous 22]. These models could be extended by incorporating the p-age, particularly, the IRRW presented here.

\section{ACKNOWLEDGMENTS}

I am grateful to Fernando A. Oliveira for the thoughtful discussions and to the National Council for Scientific and Technologic Development - CNPq, for the financial support.
[1] T. R. Malthus, An Essay on the Principle of Population (J Johnson, London, 1798)

[2] P. F. Verhulst, Correspondance Mathématique et Physique 10, 113 (1838)

[3] A. J. Lotka, Elements of physical biology (Williams and Wilkins, Baltimore, 1926)

[4] V. Voltera, Nature 118, 558 (1926)

[5] P. H. Leslie, Biometrika 33, 183 (1945)

[6] A. G. McKendrick, Proc. Edinburgh Mat. Soc. 44, 1 (1925-6)

[7] H. von Foerster, "The kinetics of cellular proliferation," (Grune and Stratten, New York, 1959) Chap. Some remarks on changing populations, pp. 382-407

[8] L. P. Lefkovitch, Biometrics 21, 1 (1965)

[9] J. VanSickle, J. Theor. Biol. 64, 571 (1977)

[10] K. Y. Lee, R. O. Barr, S. H. Gage, and A. N. Kharkar, J. Theor. Biol. 59, 33 (1976)

[11] M. Huston, D. DeAngelis, and W. Post, BioScience 38, $682(1988)$

[12] M. Kirkpatrick, Ecology 65, 1874 (1984)

[13] D. R. Clother and J. Brindley, Bull. Math. Biol. 62, 1003
(2000)

[14] R. E. Plant and L. T. Wilson, J. Math. Biol 23, 247 (1986)

[15] G. Buffoni and S. Pasquali, J. Math. Biol. 54, 555 (2007)

[16] W. J. Roltsch, F. G. Zalom, A. J. Strawn, J. F. Strand, and M. J. Pitcairn, Int J. Biometeorol 42, 169 (March 1999)

[17] R. M. Nisbet and W. S. C. Gurney, Theor. Popul. Biol. 23, 114 (1983)

[18] R. P. Feyman, R. B. Leighton, and M. Sands, "The Feyman lectures on physics," (Addison-Wesley, Reading, 1963) p. 46.1

[19] It is also possible to write an equation where $f_{k}$ is different of zero only when $k \in 0,1,2$. However, this formulation involve a negative $f_{1}$ which, besides having no probabilistic interpretation leads to numerical instabilities.

[20] J. A. R. da Cunha, A. L. A. Penna, M. H. Vainstein, R. Morgado, and F. A. Oliveira, Physics Letters A 373, 661 (2009)

[21] T. J. P. Penna, J. Stat. Phys. 78, 1629 (1995) 
[22] W. Hwang, P. L. Krapivsky, and S. Redner, Phys. Rev. Lett. 83, 1251 (1999) 